\title{
Cellular trafficking and anticancer activity of Garcinia mangostana extract-encapsulated polymeric nanoparticles
}

This article was published in the following Dove Press journal:

International Journal of Nanomedicine

6 August 2014

Number of times this article has been viewed

\author{
Porntip Pan-In ${ }^{1,2}$ \\ Supason \\ Wanichwecharungruang ${ }^{3,4}$ \\ Justin Hanes ${ }^{2,5}$ \\ Anthony J Kim ${ }^{2,6,7}$ \\ 'Program in Biotechnology, Faculty \\ of Science, Chulalongkorn University, \\ Bangkok, Thailand; ${ }^{2}$ Center for \\ Nanomedicine, Johns Hopkins \\ University School of Medicine, \\ Baltimore, MD, USA; ${ }^{3}$ Department \\ of Chemistry, Faculty of Science, \\ Chulalongkorn University, Bangkok, \\ Thailand; ${ }^{4}$ Nanotec-CU Center \\ of Excellence on Food and \\ Agriculture, Bangkok, Thailand; \\ ${ }^{5}$ Department of Ophthalmology, \\ Biomedical Engineering, Chemical \\ and Biomolecular Engineering, \\ Neurosurgery, and Oncology, \\ Johns Hopkins University School \\ of Medicine, ${ }^{6}$ Department of \\ Neurosurgery, University of Maryland \\ School of Medicine, ${ }^{7}$ Department of \\ Pharmaceutical Sciences, University \\ of Maryland School of Pharmacy, \\ Baltimore, MD, USA
}

Abstract: Garcinia mangostana Linn extract (GME) is a natural product that has received considerable attention in cancer therapy, and has the potential to reduce side effects of chemotherapeutics and improve efficacy. We formulated GME-encapsulated ethyl cellulose (GME-EC) and a polymer blend of ethyl cellulose and methyl cellulose (GME-EC/MC) nanoparticles. We achieved high drug-loading and encapsulation efficiency using a solvent-displacement method with particle sizes around $250 \mathrm{~nm}$. Cellular uptake and accumulation of GME was higher for GME-encapsulated nanoparticles compared to free GME. In vitro cytotoxicity analysis showed effective anticancer activity of GME-EC and GME-EC/MC nanoparticles in HeLa cells in a dose-dependent manner. GME-EC/MC nanoparticles showed approximately twofold-higher anticancer activity compared to GME-EC nanoparticles, likely due to their enhanced bioavailability. GME-encapsulated nanoparticles primarily entered HeLa cells by clathrin-mediated endocytosis and trafficked through the endolysosomal pathway. As far as we know, this is the first report on the cellular uptake and intracellular trafficking mechanism of drug-loaded cellulose-based nanoparticles. In summary, encapsulation of GME using cellulose-derivative nanoparticles GME-EC and GME-EC/MC nanoparticles - successfully improved the bioavailability of GME in aqueous solution, enhanced cellular uptake, and displayed effective anticancer activity.

Keywords: phytotherapy, cancer, nanoparticles, cellulose, intracellular trafficking

\section{Introduction}

Leading chemotherapeutic agents, such as paclitaxel, cisplatin, and doxorubicin, are widely used in the clinic for cancer treatment; however, systemic side effects and the development of drug resistance have limited their therapeutic efficiency. To overcome these potential problems with chemotherapeutics, a number of phytochemical and herb extracts that show anticancer activities are being widely explored for cancer therapy. ${ }^{1-5}$ Mangosteen (Garcinia mangostana Linn) is a well-known tropical fruit in Southeast Asia; xanthones from mangosteen pericarp extracts such as $\alpha$-mangostin, $\beta$-mangostin, $\gamma$-mangostin, garcinone $\mathrm{E}$, and gartanin, have received considerable interest in cancer prevention and cancer therapeutics. ${ }^{6,7}$ Several studies have reported anticancer activities of xanthones isolated from G. mangostana Linn extract (GME) in various human cancer cell lines, including those from the liver, breast, and lungs, and gastric, colorectal, and cervical cancers. ${ }^{8-13}$ These anticancer activities involve cell-cycle arrest, inhibition of cancer cell proliferation, induction of apoptosis, and inhibition of adhesion, invasion, and metastasis of tumors. ${ }^{3,6}$ However, GME's poor aqueous solubility and low bioavailability are major problems that have limited its therapeutic efficiency and pharmaceutical application. ${ }^{14}$
Correspondence: Anthony J Kim Neurosurgery and Pharmaceutical Sciences, University of Maryland, Baltimore, 655 West Baltimore Street, Baltimore, MD 2I20I, USA

Email akim@smail.umaryland.edu 
Polymeric particles are one of the most promising strategies to improve the solubility and bioavailability of poorly soluble drugs. ${ }^{15-17}$ Drug-encapsulated polymeric nanoparticles are being widely developed for cancer therapy, due to their high drug-encapsulation efficiency, passive tumor targeting, excellent endocytosis efficiency, and ability to deliver a wide range of therapeutic agents. ${ }^{15-17}$ Although pharmacological evidence of the anticancer effects of GME is growing, surprisingly there are few reported studies on the development of GME-encapsulated polymeric nanoparticles. Recently, $\alpha$-mangostin and xanthones were encapsulated in polyvinylpyrrolidone micelles and polyethylene glycolb-poly( $N$-(2-hydroxypropyl)methacrylamide-dilactate) micelles, respectively, and showed effective antitumor activity. ${ }^{18,19}$ Cellulose derivatives, such as ethyl cellulose (EC) and methyl cellulose (MC), are among the most commonly used polymers in the pharmaceutical industry, because they are biocompatible, safe, and inexpensive. ${ }^{20}$ For example, EC is a hydrophobic polymer that can encapsulate a number of hydrophobic drugs and natural substances. EC can also assemble into nanoparticles when $\mathrm{MC}$, a hydrophilic polymer, is used as a blend polymer (EC/MC) to achieve controlled release of drugs. ${ }^{20,21}$

Recently, understanding the mechanism of cellular entry and intracellular trafficking of nanoparticles has attracted a great deal of interest. ${ }^{22-24}$ Nanoparticles can enter cells via utilization of different endocytic pathways, including clathrin-mediated endocytosis, caveolae-mediated endocytosis, macropinocytosis, and other non-clathrin- and non-caveolae-mediated endocytosis. ${ }^{22-25}$ It has been reported that the cellular uptake mechanism of nanoparticles depends on the physicochemical properties of the particles, including their shape, size, surface chemistry, and particle compositions, as well as different cell types. ${ }^{24-32}$ Despite their wide use in the pharmaceutical industries, the cellular uptake and intracellular trafficking mechanism of therapeutic cellulosebased nanoparticles have not been previously reported.

In this study, we developed two GME-encapsulated cellulose derivative nanoparticles, GME-EC and GME-EC/MC, with high drug-loading and encapsulation efficiency (EE). The physicochemical properties of GME-encapsulated nanoparticles were analyzed by transmission electron microscopy (TEM), hydrodynamic particle size, and zeta-potential measurements. In vitro cytotoxicity of GME-EC and GME-EC/ MC nanoparticles against a cancer cell line (HeLa cells) was evaluated. Finally, we report cellular uptake and intracellular trafficking mechanisms of GME-encapsulated nanoparticles in HeLa cells.

\section{Materials and methods Materials}

GME with $56 \% \alpha$-mangostin (content by weight) was a generous gift from Tipco (Bangkok, Thailand). EC (viscosity 250-300 cP, ethoxy content 48\%), MC, (viscosity $400 \mathrm{cP}, 1.60 \%-1.90 \%$ degree of methoxy substitution), dialysis tubing cellulose membrane (molecular weight [MW] 12,400 Da), coumarin 6, chlorpromazine, genistein, amiloride, methyl- $\beta$-cyclodextrin, lovastatin, and all other chemicals were purchased from Sigma-Aldrich (St Louis, MO, USA). Lab-Tek ${ }^{\mathrm{TM}}$ eight-well glass-bottom tissueculture plates, minimum essential medium, fetal bovine serum, penicillin/streptomycin (pen/strep), ProLong ${ }^{\circledR}$ Gold antifade reagent, and LysoTracker ${ }^{\circledR}$ Red were from Thermo Fisher Scientific (Waltham, MA, USA).

\section{Preparation and characterization of GME-encapsulated nanoparticles}

GME-encapsulated nanoparticles (EC and blended EC/ $\mathrm{MC}$ ) were prepared by the solvent-displacement method as previously described, with a slight modification. ${ }^{21,33}$ GME and EC were dissolved in ethanol $(5 \mathrm{mg} / \mathrm{mL})$, while GME and 1:1 (w:w) EC/MC mixture was dissolved in $80 \%(\mathrm{v} / \mathrm{v})$ ethanol/water $(1 \mathrm{mg} / \mathrm{mL})$. Both mixtures $(10 \mathrm{~mL})$ were placed into a dialysis bag (cellulose membrane, MW cutoff [MWCO] $12,400 \mathrm{Da}$ ) and dialyzed against deionized (DI) water for five exchanges $(5 \times 1,000 \mathrm{~mL})$. The resulting aqueous suspension was collected, and particle morphology was characterized by TEM (H7600; Hitachi, Tokyo, Japan). Hydrodynamic particle size and zeta potential of nanoparticles were evaluated using a Zetasizer Nano ZS (Malvern Instruments, Malvern, UK).

The amount of encapsulated GME in the nanoparticles was determined by using an ultraviolet-visible (UV-vis) spectrophotometer (BioMate 3; Thermo Fisher Scientific). Briefly, $5 \mathrm{~mL}$ of each GME-encapsulated nanoparticle combination - GME-EC and GME-EC/MC - was concentrated by centrifugation at $3,000 \times \mathrm{g}$ for 20 minutes using an Amicon Ultra-15 membrane (MWCO 100,000; EMD Millipore, Billerica, MA, USA). After centrifugation, the resulting nanoparticles in the membrane were soaked in $5 \mathrm{~mL}$ of ethanol for 3 hours to extract GME from the nanoparticles. The amount of GME in the ethanol extract (absorbance at $317 \mathrm{~nm}$ ) was determined using a calibration curve. Encapsulation efficiency (EE) and loading capacity were calculated as follows:

$$
\mathrm{EE}(\%)=\frac{\text { Weight of encapsulated GME }}{\text { Weight of GME used }} \times 100
$$




$$
\text { Loading }(\%)=\frac{\text { Weight of encapsulated GME }}{\text { Weight of GME loading nanoparticles }} \times 100
$$

\section{In vitro GME release}

The release of GME from GBM-EC and GME-EC/MC nanoparticles was measured at $37^{\circ} \mathrm{C}$ in $1 \times$ phosphatebuffered saline (PBS) $\mathrm{pH}$ 7.4. The particle suspensions were spray-dried using a Büchi 190 mini spray-dryer (Büchi Labortechnik, Flawil, Switzerland). Eight milligrams of dried GME-EC and GME-EC/MC were resuspended in $20 \mathrm{~mL}$ of release medium ( $1 \times$ PBS) and then placed in a dialysis bag (MW 12,000 Da), with continuous stirring at $37^{\circ} \mathrm{C}$. A standard curve was generated from the known GME solutions prepared in the same release medium. A small aliquot of the release medium was withdrawn to analyze the amount of released GME outside the dialysis bag at $0,1,2,4,6,8$, and 24 hours, using the BioMate $3 \mathrm{UV}$-vis spectrophotometer at $320 \mathrm{~nm}$. The percentage of GME release was calculated as follows:

$$
\text { Release }(\%)=\frac{\text { Weight of released GME }}{\text { Weight of GME used }} \times 100
$$

\section{Cell culture}

Human cervical carcinoma HeLa cells were obtained from the American Type Culture Collection (Manassas, VA, USA). The cells were cultured at $37^{\circ} \mathrm{C}$ in $5 \% \mathrm{CO}_{2}$ in minimum essential medium supplemented with $10 \%$ fetal bovine serum and $1 \%$ pen/strep. For confocal microscopy, cells were seeded between $2.0 \times 10^{3}$ and $2.5 \times 10^{3}$ cells per plate onto Lab-Tek eight-well glass-bottom culture plates and incubated overnight at $37^{\circ} \mathrm{C}$. After incubation, the cell-culture medium was replaced with fresh media before nanoparticles were added.

\section{In vitro cytotoxicity study}

The cytotoxicity of free GME and GME-encapsulated nanoparticles was determined using a standard 3-(4,5dimethylthiazol-2yl)-2,5-diphenyltetrazolium bromide (MTT) assay. HeLa cells were plated onto 96-well plates at a seeding density of $1.0 \times 10^{4}$ cells per well in $200 \mathrm{~mL}$ media and incubated overnight. Our test samples were free GME dissolved in dimethyl sulfoxide (DMSO), free GME dispersed in DI water, blank EC nanoparticles, blank EC/MC nanoparticles, GME-EC, and GME-ECMC nanoparticles. The samples were diluted to $1-100 \mu \mathrm{g} / \mathrm{mL}$ with the culture medium and then added into each well and incubated for 48 hours. The equivalent amounts of DMSO, sterile DI water, and untreated cells were used as controls. After incubation, the medium was replaced with a mixture of $160 \mu \mathrm{L}$ of fresh medium and $40 \mu \mathrm{L}$ of $2 \mathrm{mg} / \mathrm{mL}$ MTT reagent and incubated at $37^{\circ} \mathrm{C}$ for 4 hours in the dark. Finally, the MTT solution was carefully removed, and $200 \mu \mathrm{L}$ of DMSO was added in each well and incubated for 30 minutes to completely dissolve the MTT formazan crystals. The absorbance was measured at $570 \mathrm{~nm}$ using the plate reader (BioMate 3 ). Cell viability $(\%)$ and half-maximal inhibitory concentration $\left(\mathrm{IC}_{50}\right)$ were then calculated for each sample.

\section{Drug uptake and accumulation in cells}

The cellular uptake and accumulation of free GME dissolved in DMSO, free GME dispersed in DI water, and GME-encapsulated nanoparticles were investigated by flow cytometry. HeLa cells were seeded onto 12 -well plates at a density of $2.0 \times 10^{5}$ cells and incubated overnight at $37^{\circ} \mathrm{C}$, $5 \% \mathrm{CO}_{2}$. The old media was replaced with $1 \mathrm{~mL}$ serum-free media with different samples at a final concentration of $5 \mu \mathrm{g} / \mathrm{mL}$. After 1 hour of incubation, the media was removed and the cells washed three times with $1 \times$ PBS to remove extracellular GME and/or nanoparticles. Then, cells were trypsinized $(0.05 \%$ trypsin-ethylenediaminetetraacetic acid [EDTA]) and resuspended in cold $1 \times$ PBS. Cell-associated fluorescence (10,000 cells per sample) was detected using a FACSCalibur ${ }^{\mathrm{TM}}$ flow cytometer (BD, Franklin Lakes, NJ, USA) to determine the mean fluorescence intensity originating from the fluorescence property of GME ( $488 \mathrm{~nm}$ excitation, $530 \pm 15 \mathrm{~nm}$ emission). Data from 10,000 events were gated using forward- and side-scatter parameters to exclude cell debris. Standard deviations were calculated from six replicates.

\section{Confocal microscopy}

An LSM 510 meta-confocal microscope (Carl Zeiss Meditec, Jena, Germany) was used to capture images. For multicolor microscopy, samples were excited with $405,488,543$, and $633 \mathrm{~nm}$ laser lines, and images were captured by multitracking to avoid bleed-through between fluorophores. All samples were maintained at $37^{\circ} \mathrm{C}$ and $5 \% \mathrm{CO}_{2}$, and observed under a 63× Plan Apo 1.4 NA oil-immersion lens (Nikon, Tokyo, Japan).

\section{Cellular trafficking of nanoparticles}

To study the cellular uptake and intracellular trafficking of nanoparticles, we encapsulated coumarin 6 , a 
hydrophobic fluorescent dye, into EC/MC (C-EC/MC) nanoparticles $(2 \% \mathrm{w} / \mathrm{w})$ instead of GME. This is because the fluorescence intensity of GME within nanoparticles was not strong enough to image them with high resolution by confocal microscopy. For the colocalization study, HeLa cells were seeded between $2.0 \times 10^{3}$ and $2.5 \times 10^{3}$ cells per plate onto Lab-Tek eight-well glass-bottom culture plates and incubated overnight at $37^{\circ} \mathrm{C}$. After overnight incubation, C-EC/MC nanoparticles were added to cells and incubated for 1, 2, and 4 hours. Cells were stained with LysoTracker ${ }^{\circledR}$ (100 nM; Thermo Fisher Scientific) for 30 minutes before imaging. After staining, cells were washed three times with $1 \times$ PBS and fixed with $2 \%$ formaldehyde and $0.2 \%$ glutaraldehyde in $1 \times$ PBS for 15 minutes. Finally, the ProLong Gold reagent with 4',6-diamidino-2-phenylindole solution was added to the sample and imaged with the LSM 510 Meta confocal microscope. The percentage of colocalization between nanoparticles and the LysoTracker was analyzed with MetaMorph $^{\circledR}$ software (Molecular Devices, Sunnyvale, CA, USA) on a per-pixel basis..$^{24,34,35}$

\section{Drug inhibition of endocytic pathway}

To determine the mechanism of cellular entry for GME-EC/MC nanoparticles, HeLa cells were plated at a seeding density of $2.0 \times 10^{5}$ cells/well in 12 -well plates in serum-free media. Prior to GME-EC/MC nanoparticle addition, serum-free media with different endocytic pathway inhibitors, chlorpromazine $(10 \mu \mathrm{g} / \mathrm{mL})$, sucrose $(0.45 \mathrm{M})$, and genistein $(200 \mu \mathrm{M})$ were added to the cells and incubated for 30 minutes, followed by the addition of nanoparticles. For methyl- $\beta$-cyclodextrin $(10 \mathrm{mM})$ and lovastatin $(1 \mu \mathrm{g} / \mathrm{mL})$, cells were treated for $15 \mathrm{~min}$ utes, and then nanoparticles were added and incubated for 30 minutes. For $4^{\circ} \mathrm{C}$ samples, they were chilled for 30 minutes before the addition of nanoparticles and maintained at $4{ }^{\circ} \mathrm{C}$ during the incubation period (1 hour). After incubation, all samples were washed three times with $1 \times$ PBS to remove extracellular nanoparticles. Cells were harvested with $0.05 \%$ trypsin-EDTA and resuspended with cold $1 \times$ PBS. Cell-associated fluorescence $(10,000$ cells per sample) was detected using the FACSCalibur flow cytometer to determine the mean fluorescence intensity originating from the fluorescence property of GME (488 nm excitation, $530 \pm 15 \mathrm{~nm}$ emission). Data from 10,000 events were gated using forward- and side-scatter parameters to exclude cell debris. Standard deviations were calculated from three replicates.

\section{Statistical analysis}

Statistical analysis of data was performed using a two-tailed Student's $t$-test or one-way analysis of variance with Tukey's honest significant difference test using SPSS 18.0 (SPSS Inc., Chicago, IL, USA). Differences were considered to be statistically significant at a level of $P<0.05$.

\section{Results and discussion Characterization of nanoparticles}

We encapsulated GME into cellulose-derivative polymeric nanoparticles - GME-EC and GME-EC/MC - using a solvent-displacement method. We formulated GME-EC/MC nanoparticles at a lower polymer concentration compared to GME-EC nanoparticles, since the MC chains were highly water-soluble and contributed to the swelling of EC/MC nanoparticles. ${ }^{21,33}$ Both GME-EC and GME-EC/MC nanoparticles achieved high drug-loading efficiency and EE. We achieved an EE of $86.2 \% \pm 3.9 \%$ and $87.4 \% \pm 4.1 \%$ at GME loading of $46.7 \% \pm 2.2 \%$ and $46.7 \% \pm 2.2 \%$ for GME-EC and GME-EC/MC nanoparticles, respectively (Table 1). GME is a hydrophobic molecule that is suitable for efficient encapsulation into cellulose-derivative nanoparticles. ${ }^{18,19}$ The morphology of GME-encapsulated nanoparticles was confirmed by TEM, which showed spherical shapes for both types of nanoparticles (Figure 1).

Dynamic light-scattering analysis showed that the hydrodynamic diameters of the GME-EC and GME-EC/MC nanoparticles were $253.3 \pm 8.6 \mathrm{~nm}$ and $249.2 \pm 13.1 \mathrm{~nm}$, respectively (Table 1). The zeta potential of the GME-EC and GME-EC/MC nanoparticles was $-30.9 \pm 0.4 \mathrm{mV}$ and $-11.7 \pm 0.5 \mathrm{mV}$, respectively (Table 1 ). As ethanol was slowly displaced by water, the polymeric chains in EC likely self-assembled into spherical nanoparticles with the hydroxyl groups of the sugar units arranged outside the nanoparticles, while the ethoxy portions oriented away from the surrounding water. This arrangement aided nanoparticles to suspend in water, and at the same time encapsulated hydrophobic GME within the core of nanoparticles. ${ }^{21,33}$ For $\mathrm{EC} / \mathrm{MC}$ nanoparticles, the MC chains likely entangled with the EC chains as the nanoparticles were formed. Therefore, the shell of EC/MC nanoparticles would have been composed of an EC/MC polymer blend. We concluded that GME-encapsulated nanoparticles improved the bioavailability of GME.

\section{In vitro cytotoxicity to cancer cells}

The anticancer activity of GME-encapsulated nanoparticles, free GME dissolved in DMSO or in water, and blank EC and 
Table I Physicochemical properties, drug-encapsulation efficiency (EE), and drug-loading capacity of GME-encapsulated nanoparticles

\begin{tabular}{llllll}
\hline Formulation & Diameter $(\mathbf{n m})^{\mathrm{a}}$ & PDI $^{\mathrm{b}}$ & ${\text { S-potential }(\mathbf{m V})^{\mathrm{c}}}$ & EE (\%) & Loading (\%) \\
\hline GME-EC & $253.3 \pm 8.6$ & $0.05 \pm 0.01$ & $-30.9 \pm 0.4$ & $86.2 \pm 3.9$ & $43.1 \pm 1.9$ \\
GME-EC/MC & $249.2 \pm 13.1$ & $0.07 \pm 0.04$ & $-11.7 \pm 0.5$ & $87.5 \pm 4.1$ & $43.5 \pm 2.1$ \\
C-EC & $239.9 \pm 16.9$ & $0.12 \pm 0.04$ & $-21.7 \pm 1.9$ & - & - \\
C-EC/MC & $233.8 \pm 8.1$ & $0.12 \pm 0.02$ & $-18.8 \pm 2.2$ & - & - \\
\hline
\end{tabular}

Notes: ${ }^{a}$ Diameter (number mean) measured by dynamic light scattering; data represent the average of three independent experiments \pm SD; b the polydispersity index (PDI), measured by dynamic light scattering, represents relative variance in particle-size distribution; a PDI of I indicates a large distribution of particle size, whereas a PDI of 0 indicates a monodisperse size distribution; data represent the average of three independent experiments \pm SD; ${ }^{c}$ measured at $\mathrm{pH} 7.1$; data represent the average of three independent experiments \pm SD.

Abbreviations: GME, Garcinia mangostana Linn extract; EC, ethyl cellulose; MC, methyl cellulose; SD, standard deviation; C, coumarin 6.

EC/MC nanoparticles were tested using the MTT assay. Both blank EC and blank EC/MC nanoparticles did not show any cytotoxic activities at the concentrations that we tested (Figure 2 and Table 2). This showed the biocompatible and nontoxic nature of our cellulose-based nanoparticles. Free GME dissolved in DMSO or in water and GME-encapsulated nanoparticles (GME-EC and GME-EC/MC nanoparticles) showed a dose-dependent cytotoxicity in HeLa cells (Figure 2A). The order of cytotoxic activities $\left(\mathrm{IC}_{50}\right)$ was: free GME dissolved in DMSO $\left(\mathrm{IC}_{50} 7.33 \mu \mathrm{g} / \mathrm{mL}\right)>$ GME-EC/MC nanoparticles $\left(\mathrm{IC}_{50} 7.43\right)>$ GME-EC nanoparticles $\left(\mathrm{IC}_{50} 16.17\right)>$ free GME dissolved in water $\left(\mathrm{IC}_{50} 23.09\right)$ (Table 2). Low cytotoxicity observed from free GME dissolved in water was not surprising, since GME has poor solubility in water, which limits its activity. In contrast, both GME-encapsulated nanoparticles that were dispersed in water - GME-EC and GME-EC/MC nanoparticles - showed significantly higher cytotoxic activities compared to free GME dissolved in water. These results indicate that the encapsulation of GME into a polymeric nanoparticle platform improved the bioavailability of GME.

GME-EC/MC nanoparticles showed approximately twofold-higher cytotoxicity in HeLa cells compared to
GME-EC nanoparticles. This result is in good agreement with previous reports of Suwannateep et al where encapsulation of curcumin in EC/MC blend nanoparticles showed higher cytotoxicity compared to curcumin-encapsulated EC nanoparticles. ${ }^{21,33}$ The in vitro release rate of GME from GBM-EC and GME-EC/MC nanoparticles was comparable, with no statistically significant differences (Figure 3). These results indicated that the difference in $\mathrm{IC}_{50}$ between GME-EC and GME-EC/MC nanoparticles was not due to the difference in their GME release rates after 24 hours. Our results show that different types of polymer compositions of nanoparticles can significantly affect the in vitro cytotoxicity of GME. Interestingly, at the highest GME concentration that we tested, $100 \mu \mathrm{g} / \mathrm{mL}$, both GME-EC and GME-EC/MC nanoparticles showed higher cytotoxicity than free GME dissolved in DMSO (Figures 2B and S1).

\section{Cellular uptake and endocytic pathway of nanoparticles}

Since GME-EC/MC nanoparticles showed the most promising anticancer activity, we investigated cellular uptake and intracellular trafficking using this nanoparticle formulation.
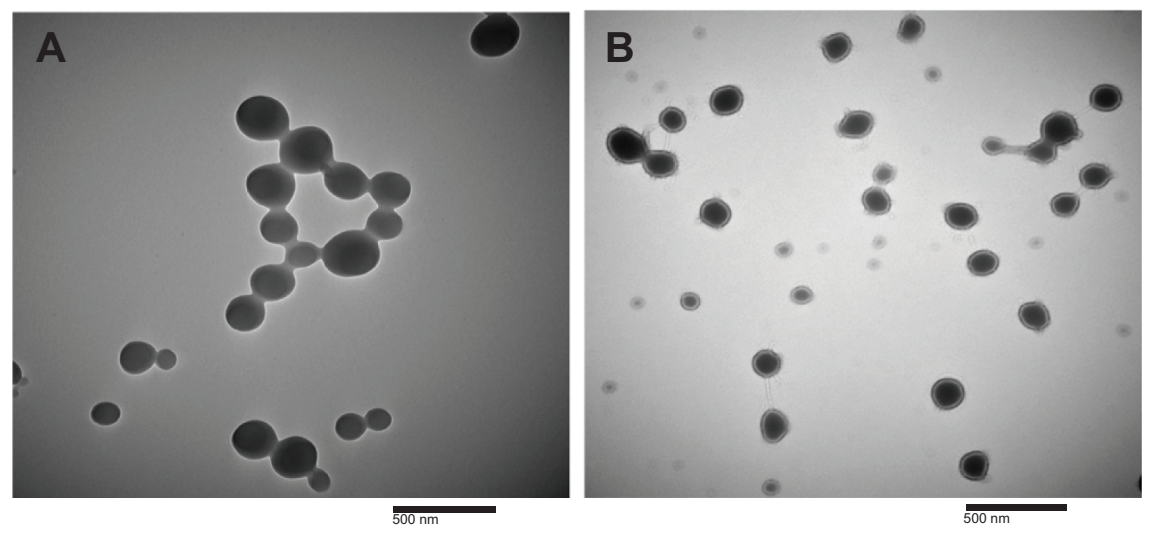

Figure I Transmission electron microscopy images of (A) GME-encapsulated EC nanoparticles and (B) GME-encapsulated EC/MC nanoparticles at 50,000× magnification. Note: The scale bar denotes $500 \mathrm{~nm}$.

Abbreviations: GME, Garcinia mangostana Linn extract; EC, ethyl cellulose; MC, methyl cellulose. 


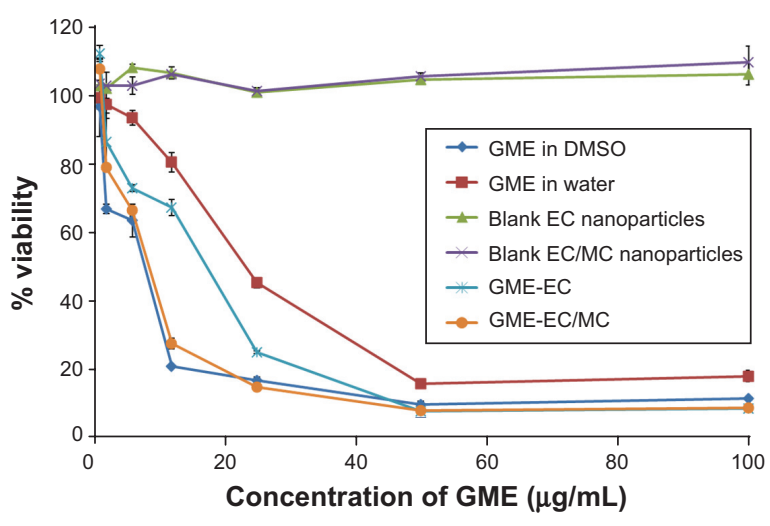

Figure 2 Cytotoxic activity in HeLa cells using different formulations of free GME and GME-encapsulated nanoparticles.

Notes: Cell viability was evaluated by MTT assay. Data represent means \pm standard deviation of three separate experiments $(n=3)$.

Abbreviations: GME, Garcinia mangostana Linn extract; DMSO, dimethyl sulfoxide; EC, ethyl cellulose; MC, methyl cellulose; MTT, 3-(4,5-dimethylthiazol-2-yl)-2,5diphenyltetrazolium bromide.

GME-EC/MC nanoparticles showed significantly higher cellular uptake in HeLa cells compared to free GME dissolved in DI water or in DMSO (Figure 4). These results suggest that the encapsulation of GME in cellulose-derivative nanoparticles is an effective strategy to enhance the cellular uptake and accumulation of GME. As discussed earlier, the cytotoxicity of GME-EC/MC nanoparticles was comparable to free GME dissolved in DMSO, which was likely due to slow and sustained release of encapsulated GME from GME-EC/MC nanoparticles. Therefore, combined with enhanced cellular uptake and accumulation, GMEencapsulated nanoparticles may provide a better alternative for GME cancer therapy.

To determine the endocytic pathway of GME-encapsulated nanoparticles, we measured the uptake of GME-EC/MC nanoparticles in HeLa cells treated with different endocytic pathway inhibitors, using flow cytometry. Compared to the untreated control cells, the uptake of GME-EC/MC nanoparticles was significantly reduced $(P<0.05)$ following treatment of cells with chlorpromazine, which blocks clathrinmediated endocytosis by causing clathrin to accumulate in late endosomes (Figure 5A). Similarly, hypertonic sucrose

Table $2 \quad I_{50}$ values for free GME and GME-encapsulated nanoparticles

\begin{tabular}{ll}
\hline Formulation & IC $_{50}(\mu \mathbf{g} / \mathbf{m L})$ \\
\hline GME in DMSO & 7.33 \\
GME in water & 23.09 \\
GME-EC & 16.17 \\
GME-EC/MC & 7.43 \\
\hline
\end{tabular}

Abbreviations: GME, Garcinia mangostana Linn extract; DMSO, dimethyl sulfoxide; EC, ethyl cellulose; $\mathrm{MC}$, methyl cellulose; $\mathrm{IC}_{50}$, half-maximal inhibitory concentration.

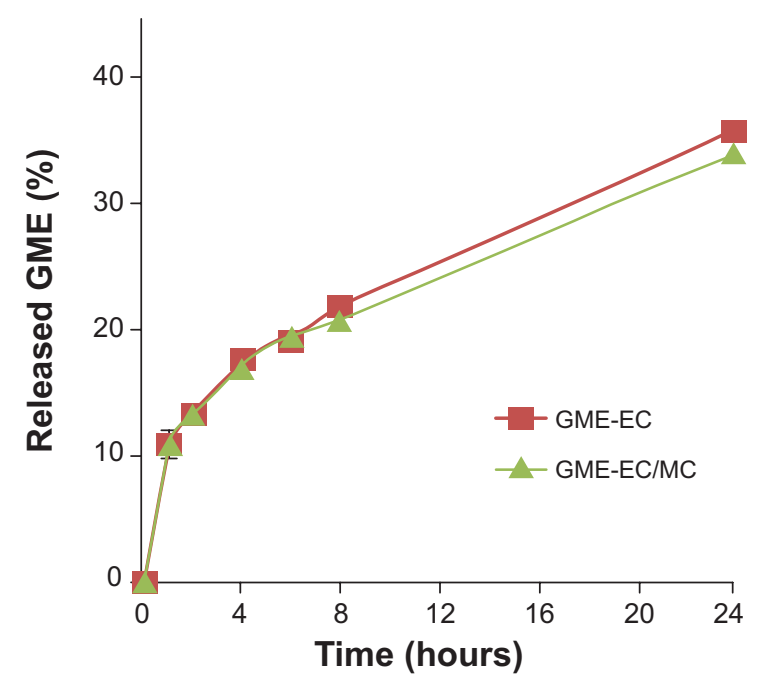

Figure 3 Cumulative in vitro release of GME from GME-EC and GME-EC/MC nanoparticles in IX PBS ( $\mathrm{pH} 7.4)$.

Note: Data represent means \pm standard deviation of three separate experiments $(n=3)$. Abbreviations: GME, Garcinia mangostana Linn extract; EC, ethyl cellulose; MC, methyl cellulose; PBS, phosphate-buffered saline.

$(0.45 \mathrm{M})$, which inhibits clathrin-mediated endocytosis, also reduced the cellular uptake of GME-EC/MC nanoparticles ( $\sim 23 \%$ reduction). In contrast, the cellular uptake of GME-EC/MC nanoparticles was not affected by genistein or amiloride, inhibitors of caveolae-mediated uptake and macropinocytosis, respectively (Figure 5, B and D). In addition, the uptake of GME-EC/MC nanoparticles was significantly inhibited when the cells were incubated at $4{ }^{\circ} \mathrm{C}$, confirming that they entered HeLa cells via energy-dependent endocytosis and not via direct penetration of plasma membrane (Figure 5C). The cellular uptake of GME-EC/ MC nanoparticles was significantly reduced $(P<0.05)$ by cholesterol depletion, using methyl-b-cyclodextrin (extracts

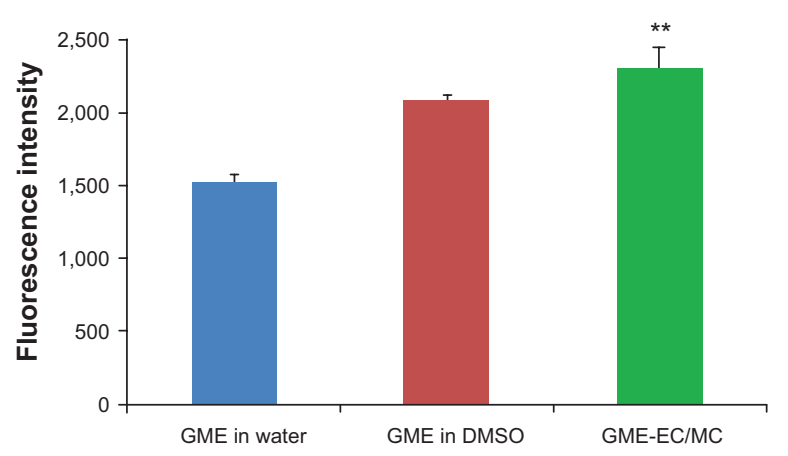

Figure 4 Cellular uptake and accumulation of free GME and GME-encapsulated nanoparticles in HeLa cells.

Notes: Data represent means \pm standard deviation of three separate experiments $(n=3)$. Statistical analysis was performed using analysis of variance with Tukey's test. $* * P<0.05$

Abbreviations: GME, Garcinia mangostana Linn extract; DMSO, dimethyl sulfoxide; $\mathrm{EC}$, ethyl cellulose; MC, methyl cellulose. 

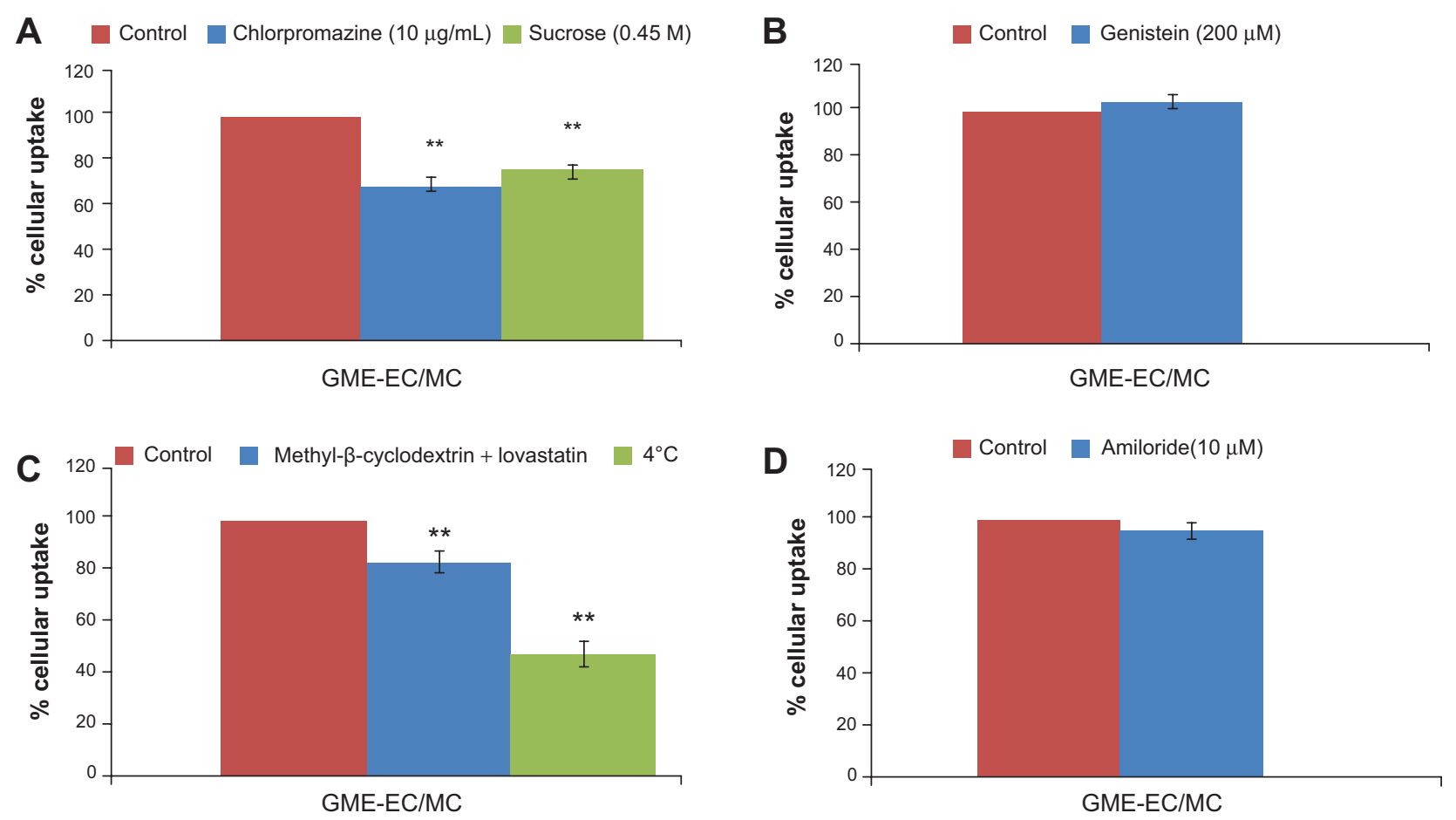

Figure 5 Endocytic mechanism of GME-EC/MC nanoparticles in HeLa cells. Cells were treated with various endocytic inhibitors prior to nanoparticle addition.

Notes: (A) Chlorpromazine and sucrose $0.45 \mathrm{M}$, inhibitors of clathrin-mediated endocytosis; (B) genistein, an inhibitor of caveolae-mediated endocytosis; (C) methyl- $\beta$ cyclodextrin and lovastatin, which depleted cholesterol, and uptake of nanoparticles at $4^{\circ} \mathrm{C}$; and (D) amiloride, an inhibitor of macropinocytosis. $* * P<0.05$ compared to no-treatment control, calculated using two-tailed Student's $t$-test.

Abbreviations: GME, Garcinia mangostana Linn extract; EC, ethyl cellulose; MC, methyl cellulose.

cholesterol from plasma membrane) and lovastatin (inhibitor of de novo cholesterol synthesis), in good agreement with the cholesterol-dependent nature of clathrin-mediated endocytosis (Figure 5C). ${ }^{36-39}$ Together, these findings suggest that GME-EC/MC nanoparticles entered HeLa cells primarily via clathrin-mediated endocytosis.

\section{Intracellular trafficking of nanoparticles}

The general fate of most nanoparticles following clathrinmediated endocytosis is the endolysosomal pathway, where early endosomes mature into late endosomes, and finally to lysosomes. ${ }^{40}$ To determine whether EC/MC nanoparticles were trafficked via the endolysosomal pathway, we performed colocalization studies using the LysoTracker, a well-characterized marker for late endosomes and lysosomes. The hydrodynamic size and zeta potential of C-EC/ $\mathrm{MC}$ nanoparticles were similar to GME-EC/MC nanoparticles (Table 1). Confocal microscopy images showed a high degree of colocalization between $\mathrm{C}$-EC/MC nanoparticles and the LysoTracker within 1 hour (Figure 6), which continued to increase significantly until the 2-hour time point (Figure 6). Interestingly, the degree of colocalization between $\mathrm{C}$-EC/MC nanoparticles and the LysoTracker decreased at the 4-hour time point (Figure 6), which suggests that $\mathrm{C}$-EC/MC nanoparticles were providing controlled release of drugs once the particles were entrapped in the acidic vesicles. This is in good agreement with previous reports, where curcumin-encapsulated EC/MC nanoparticles showed faster drug release at lower $\mathrm{pH}$, compared to physiological $\mathrm{pH} .{ }^{21,33}$ These results indicate that EC/MC nanoparticles traffic through the endolysosomal pathway following their cellular entry via clathrin-mediated endocytosis.

\section{Conclusion}

In this study, GME (with 56\% $\alpha$-mangostin content) was successfully encapsulated into cellulose-derivative nanoparticles - GME-EC and GME-EC/MC nanoparticles with high $\mathrm{EE}(\sim 87 \%)$ and loading capacity ( 46\%). GME-EC and GME-EC/MC nanoparticles showed spherical morphology and a particle size around $250 \mathrm{~nm}$ with surface charges of $-30.9 \mathrm{mV}$ and $-11.7 \mathrm{mV}$, respectively. GME-EC and GME-EC/MC nanoparticles showed effective anticancer activity in HeLa cells, and enhanced the cellular uptake of encapsulated GME compared to free GME. GME-encapsulated nanoparticles primarily entered HeLa cells by clathrin-mediated 
A
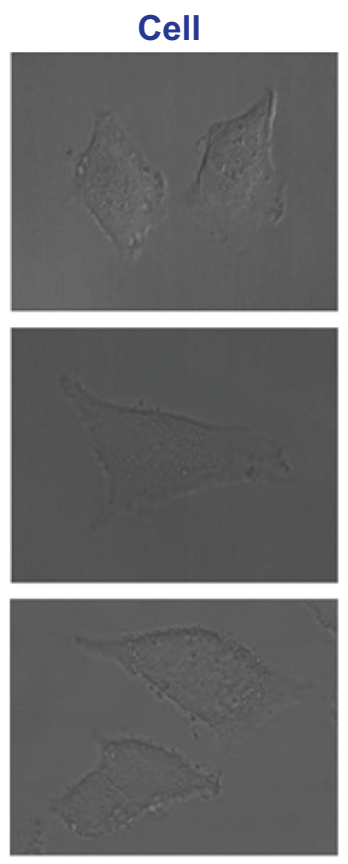

C-EC/MC
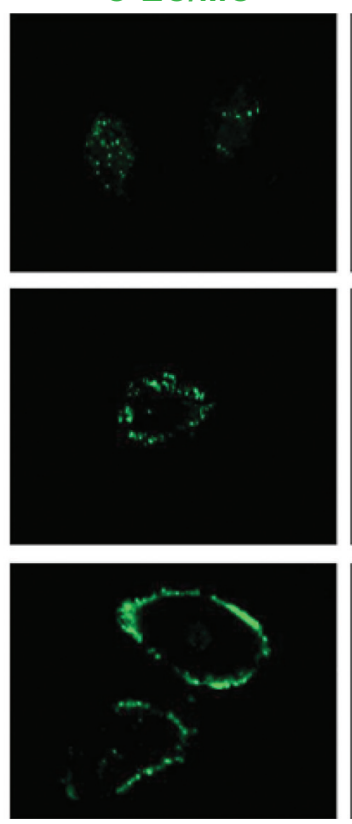
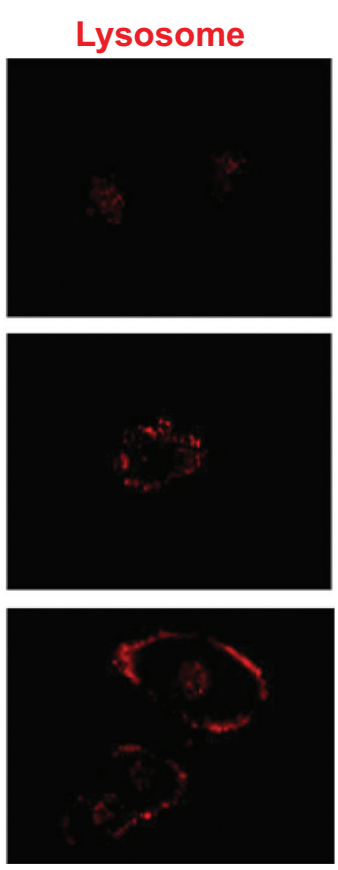

Merge
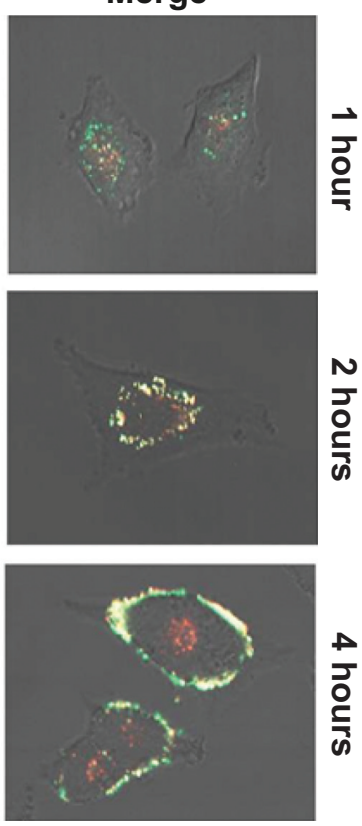

\section{B}

After 1 hour

After 2 hours

After 4 hours

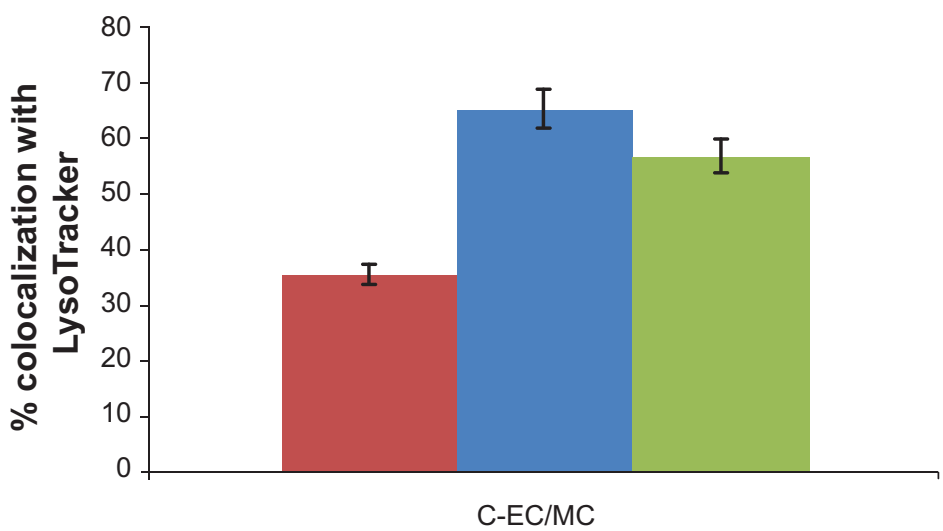

Figure 6 Representative confocal microscopy images of (A) C-EC/MC nanoparticles (green) with LysoTracker (red) in HeLa cells. The colocalization between C-EC/MC nanoparticles and LysoTracker are shown in the merged images (yellow). (B) Quantification of colocalization analysis at different time points.

Abbreviations: EC, ethyl cellulose; MC, methyl cellulose; C, coumarin 6.

endocytosis and were trafficked through the endolysosomal pathway. Our results show that GME-encapsulated cellulosederivative nanoparticles, especially GME-EC/MC nanoparticles, have the potential to provide a better alternative strategy for cancer therapy.

\section{Acknowledgments}

The authors thank the Royal Golden Jubilee PhD Program (PHD0075/2550), the Nanotechnology Center (NANOTEC), NSTD, Ministry of Science and Technology, Thailand and Rachadapisaek Sompote Fund Post-Doctoral Support,
Pharmaceutical Research and Manufacturers of America (PhRMA) Foundation Research Starter Grant, and the National Heart, Lung, and Blood Institute (F32L103137) at the National Institute of Health for their financial support. We thank Ms Varapon Rakkhitawatthana for $\mathrm{IC}_{50}$ calculations and Dr Himat Patel and Dr Craig Schneider for helpful comments. We also thank the Wilmer Microscopy and Imaging Core Facility at Johns Hopkins School of Medicine.

\section{Disclosure}

The authors report no conflicts of interest in this work. 


\section{References}

1. Nishino H, Murakoshi M, Mou XY, et al. Cancer prevention by phytochemicals. Oncology. 2005;69:38-40.

2. Nishino H, Satomi Y, Tokuda H, Masuda M. Cancer control by phytochemicals. Curr Pharm Des. 2007;13:3394-3399.

3. Akao Y, Nakagawa Y, Iinuma M, Nozawa Y. Anti-cancer effects of xanthones from pericarps of mangosteen. Int J Mol Sci. 2008;9:355-370.

4. Bar-Sela G, Epelbaum R, Schaffer M. Curcumin as an anti-cancer agent: review of the gap between basic and clinical applications. Curr Med Chem. 2010;17:190-197.

5. Thomasset SC, Berry DP, Garcea G, Marczylo T, Steward WP, GescherAJ. Dietary polyphenolic phytochemicals - promising cancer chemopreventive agents in humans? A review of their clinical properties. Int J Cancer. 2007; 120:451-458.

6. Pedraza-Chaverri J1, Cárdenas-Rodríguez N, Orozco-Ibarra M, Pérez-Rojas JM. Medicinal properties of mangosteen (Garcinia mangostana). Food Chem Toxicol. 2008;46:3227-3239.

7. Shan T1, Ma Q, Guo K, et al. Xanthones from mangosteen extracts as natural chemopreventive agents: potential anticancer drugs. Curr Mol Med. 2011;11:666-677.

8. Shadid KA, Shaari K, Abas F, et al. Cytotoxic caged-polyprenylated xanthonoids and a xanthone from Garcinia cantleyana. Phytochemistry. 2007;68:2537-2544.

9. Matsumoto K, Akao Y, Kobayashi E, et al. Induction of apoptosis by xanthones from mangosteen in human leukemia cell lines. J Nat Prod. 2003;66:1124-1127.

10. Moongkarndi P, Kosem N, Kaslungka S, Luanratana O, Pongpan N, Neungton N. Antiproliferation, antioxidation and induction of apoptosis by Garcinia mangostana (mangosteen) on SKBR3 human breast cancer cell line. J Ethnopharmacol. 2004;90:161-166.

11. Matsumoto K, Akao Y, Ohguchi K, et al. Xanthones induce cell-cycle arrest and apoptosis in human colon cancer DLD-1 cells. Bioorg Med Chem. 2005;13:6064-6069.

12. Suksamrarn S, Komutiban O, Ratananukul P, Chimnoi N, Lartpornmatulee N, Suksamrarn A. Cytotoxic prenylated xanthones from the young fruit of Garcinia mangostana. Chem Pharm Bull (Tokyo). 2006;54: 301-305

13. Ho CK, Huang YL, Chen CC. Garcinone E, a xanthone derivative, has potent cytotoxic effect against hepatocellular carcinoma cell lines. Planta Med. 2002;68:975-979.

14. Li L, Brunner I, Han AR, et al. Pharmacokinetics of $\alpha$-mangostin in rats after intravenous and oral application. Mol Nutr Food Res. 2011;55 Suppl 1:S67-S74.

15. Brannon-Peppas L, Blanchette JO. Nanoparticle and targeted systems for cancer therapy. Adv Drug Deliv Rev. 2004;56:1649-1659.

16. Brigger I, Dubernet C, Couvreur P. Nanoparticles in cancer therapy and diagnosis. Adv Drug Deliv Rev. 2002;54:631-651.

17. Peer D, Karp JM, Hong S, Farokhzad OC, Margalit R, Langer R. Nanocarriers as an emerging platform for cancer therapy. Nat Nanotechnol. 2007;2:751-760.

18. Aisha AF, Ismail Z, Abu-Salah KM, Majid AM. Solid dispersions of $\alpha$-mangostin improve its aqueous solubility through self-assembly of nanomicelles. J Pharm Sci. 2012;101:815-825.

19. Khonkarn R, Mankhetkorn S, Talelli M, Hennink WE, Okonogi S. Cytostatic effect of xanthone-loaded mPEG-b-p(HPMAm-Lac2) micelles towards doxorubicin sensitive and resistant cancer cells. Colloids Surf B Biointerfaces. 2012;94:266-273.

20. Duarte AR, Gordillo MD, Cardoso MM, Simplicio AL, Duarte CM. Preparation of ethyl cellulose/methyl cellulose blends by supercritical antisolvent precipitation. Int J Pharm. 2006;311:50-54.
21. Suwannateep N, Banlunara W, Wanichwecharungruang SP, Chiablaem K, Lirdprapamongkol K, Svasti J. Mucoadhesive curcumin nanospheres: biological activity, adhesion to stomach mucosa and release of curcumin into the circulation. J Control Release. 2011;151:176-182.

22. Sahay G, Alakhova DY, Kabanov AV. Endocytosis of nanomedicines. J Control Release. 2010;145:182-195.

23. Kim A, Hanes J. The emergence of multiple particle tracking in intracellular trafficking of nanomedicines. Biophys Rev. 2012;4:83-92.

24. Kim AJ, Boylan NJ, Suk JS, Lai SK, Hanes J. Non-degradative intracellular trafficking of highly compacted polymeric DNA nanoparticles. J Control Release. 2012;158:102-107.

25. Sandvig K, Grimmer S, Iversen TG, et al. Ricin transport into cells: studies of endocytosis and intracellular transport. Int J Med Microbiol. 2000;290:415-420.

26. Chung TH, Wu SH, Yao M, et al. The effect of surface charge on the uptake and biological function of mesoporous silica nanoparticles in 3T3-L1 cells and human mesenchymal stem cells. Biomaterials. 2007; 28:2959-2966.

27. Martins S, Costa-Lima S, Carneiro T, Cordeiro-da-Silva A, Souto EB, Ferreira DC. Solid lipid nanoparticles as intracellular drug transporters: an investigation of the uptake mechanism and pathway. Int J Pharm. 2012;430:216-227.

28. Gratton SE, Ropp PA, Pohlhaus PD, et al. The effect of particle design on cellular internalization pathways. Proc Natl Acad Sci US A. 2008;105: 11613-11618.

29. Dausend J, Musyanovych A, Dass M, et al. Uptake mechanism of oppositely charged fluorescent nanoparticles in HeLa cells. Macromol Biosci. 2008;8:1135-1143.

30. Verma A, Stellacci F. Effect of surface properties on nanoparticle-cell interactions. Small. 2010;6:12-21.

31. He C, Hu Y, Yin L, Tang C, Yin C. Effects of particle size and surface charge on cellular uptake and biodistribution of polymeric nanoparticles. Biomaterials. 2010;31:3657-3666.

32. Rejman J, Oberle V, Zuhorn IS, Hoekstra D. Size-dependent internalization of particles via the pathways of clathrin- and caveolae-mediated endocytosis. Biochem J. 2004;377:159-169.

33. Suwannateep N, Wanichwecharungruang S, Fluhr J, Patzelt A, Lademann J, Meinke MC. Comparison of two encapsulated curcumin particular systems contained in different formulations with regard to in vitro skin penetration. Skin Res Technol. 2013;19:1-9.

34. Lai SK, Hida K, Man ST, et al. Privileged delivery of polymer nanoparticles to the perinuclear region of live cells via a non-clathrin, nondegradative pathway. Biomaterials. 2007;28:2876-2884.

35. Boylan NJ, Kim AJ, Suk JS, et al. Enhancement of airway gene transfer by DNA nanoparticles using a pH-responsive block copolymer of polyethylene glycol and poly-L-lysine. Biomaterials. 2012; 33:2361-2371.

36. Ohtani Y, Irie T, Uekama K, Fukunaga K, Pitha J. Differential effects of alpha-, beta- and gamma-cyclodextrins on human erythrocytes. Eur J Biochem. 1989;186:17-22.

37. Rodal SK, Skretting G, Garred O, Vilhardt F, van Deurs B, Sandvig K. Extraction of cholesterol with methyl-beta-cyclodextrin perturbs formation of clathrin-coated endocytic vesicles. Mol Biol Cell. 1999; 10:961-974.

38. Pichler H, Riezman H. Where sterols are required for endocytosis. Biochim Biophys Acta. 2004;1666:51-61.

39. Tobert JA. Lovastatin and beyond: the history of the HMG-CoA reductase inhibitors. Nat Rev Drug Discov. 2003;2:517-526.

40. Pillay CS, Elliott E, Dennison C. Endolysosomal proteolysis and its regulation. Biochem J. 2002;363:417-429. 


\section{Supplementary material}

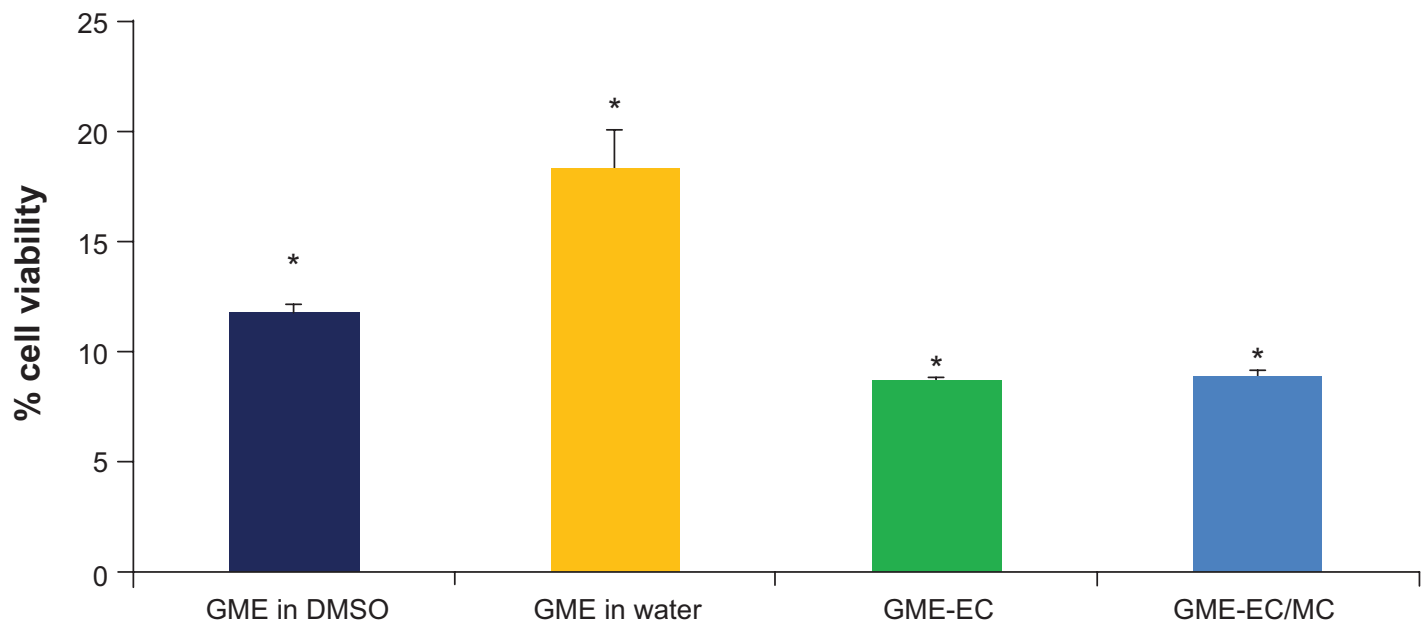

Concentration $100 \mu \mathrm{g} / \mathrm{mL}$

Figure SI Cytotoxic activity of different formulation of $100 \mu \mathrm{g} / \mathrm{mL}$ free GME and GME-encapsulated nanoparticles in HeLa cells.

Notes: Data represent means \pm standard deviation $(n=3)$. $* P<0.05$. Statistical analysis was performed by analysis of variance with Tukey's honest significant difference test. Abbreviations: GME, Garcinia mangostana Linn extract; EC, ethyl cellulose; MC, methyl cellulose; DMSO, dimethyl sulfoxide.

\section{Publish your work in this journal}

The International Journal of Nanomedicine is an international, peerreviewed journal focusing on the application of nanotechnology in diagnostics, therapeutics, and drug delivery systems throughout the biomedical field. This journal is indexed on PubMed Central, MedLine, CAS, SciSearch ${ }^{\circledR}$, Current Contents ${ }^{\circledR} /$ Clinical Medicine,
Journal Citation Reports/Science Edition, EMBase, Scopus and the Elsevier Bibliographic databases. The manuscript management system is completely online and includes a very quick and fair peer-review system, which is all easy to use. Visit http://www.dovepress.com/ testimonials.php to read real quotes from published authors. 